\title{
Zero in on Postpartum Hemorrhage to Reduce Cuba's Maternal Mortality
}

\author{
Sonia Águila MD MS
}

Postpartum hemorrhage (PPH) is the most frequent cause of severe maternal morbidity (SMM) and the first direct cause of maternal death in most countries. In Africa and Asia, it accounts for about one third of all maternal deaths. Put more graphically: worldwide, one woman dies every minute from $\mathrm{PPH}$. Defined as blood loss of $\geq 500 \mathrm{~mL}$ after vaginal birth or $\geq 1000 \mathrm{~mL}$ after cesarean delivery, PPH can be fatal in just two hours.

In Cuba, between 2000 and 2012, maternal deaths directly related to obstetric causes totaled $410,24.1 \%$ of which occurred postpartum, with PPH the leading cause.[1] While Cuba is among the Latin American countries with lowest maternal mortality, the decline has been slow over the last 20 years: in 1998, direct maternal mortality was 26.5 per 100,000 live births and in 2012 , the rate was 21.5 . This is troubling and deserves careful study, especially given that Cuba has a single, unified health system supported by significant political will-determining factors in important advances made in maternal-child health on par with wealthier countries.

Years of experience, enriched by exchanges with young physicians, case discussions concerning SMM and fatalities caused by $\mathrm{PPH}$, as well as service audits carried out across Cuba, have led me to several conclusions on risk of death from PPH and how to effectively mitigate that risk. I share some of this thinking here, hoping it will contribute to efforts to further reduce maternal mortality.

\section{Frequent errors in clinical assessment}

- Upon diagnosis, extent/amount of bleeding underestimated (in up to $50 \%$ of cases)

- Failure to identify-during either antenatal period or deliveryrisk factors for bleeding that can produce uterine atony, such as: uterine fiber fatigue secondary to prolonged labor or excessively rapid delivery; or inability of uterine fibers to contract, after overdistension from multiple gestation, fetal macrosomy, polyhydramnios or fatty infiltration of the myometrium due to obesity

- Excessive use of oxytocins to induce or manage labor

Essential best practices to reduce PPH and resulting mortality

- The overall approach should be guided by a vision that is both interdisciplinary and preventive.

- From preconception forward: protocols and clinical guidelines should detail all events that constitute "risk of bleeding."

- During antenatal care: interrelationships among family physicians, obstetricians and participating nutritionists should be well defined, to work harmoniously and avoid patient anxiety.

- For those at risk of bleeding: patients should reach term with optimal hematocrit and hemoglobin levels, to better withstand postpartum bleeding.

-When bleeding occurs: obstetricians and perinatologists should not be the only ones involved in patient management; rather, this should also be the purview of obstetric nurses, anesthesiologists, internists, hematologists and those in other fields whose participation and knowledge are vital to the attending physician, before the patient's clinical state becomes severe.

- For obstetrical services: the gold standard should be quality and rigorous adherence to protocols for labor and delivery.

- For cesarean sections: strict control of protocols should be observed, including criteria for indicating cesareans, risk of bleeding according to diagnosis, anesthesiologist evaluation, quality of the surgical team, a well-performed and smooth surgical technique, and excellence throughout the procedure.

- At delivery: the entire protocol for active birthing management must be applied, including oxytocin administration as the infant is being born, plus gentle and maintained placenta traction and uterine massage for two to four hours after delivery.

- PPH emergencies: chiefs and their deputies on call in obstetric services must be fully proficient in emergency procedures for $\mathrm{PPH}$ management, such as total and partial hysterectomy and hypogastric artery ligature.

- First postpartum hour: this is the "golden hour," when the patient's prognosis and survival are determined. In this period, not only should cause of bleeding be established, but application of a specific therapy should also be well advanced.

- Postpartum "golden minutes": by this crucial time, treatment strategy and organization should have been defined, according to guidelines established by the service for such cases, including initial measures, each team member's responsibilities, and support from related professionals, as well as the role of the hospital's experienced Severe Case Commission.[2]

- Specialized training: capacity-building needs should be identified in all services for pregnant women and new mothers, not only for physicians, but also for nurses, technical staff, etc.

- Specific competencies: physicians should be trained in opportune diagnosis of PPH, based not only on extent of bleeding, but also on very early signs of acute peripheral hypoperfusion.

Regularly programmed hospital activities to ensure best practices

- Training at different levels in various services, using simulators when necessary

- Competency and performance reviews for all personnel in obstetric and nursing services

- Frequent audits of services where obstetric patients are managed: urgent care facilities, labor and delivery suites, operating rooms, surgical recovery and other areas where emergency cases may present

Taken together and applied nationally, these actions will allow us to control morbidity-and thus reduce maternal mortalitycaused by PPH. - -1/

1. Cabezas Cruz E. Evolución de la mortalidad materna en Cuba. Rev Cubana Salud Pública. 2006 Jan-Mar;32(1). Spanish.

2. Águila Setién S. Una estrategia para la disminución de la mortalidad materna. Rev Cubana Obstet Ginecol. 2012 Apr-Jun;38(2):281-9. Spanish.

Submitted: April 8, 2014

Approved for publication: December 22, 2014

Disclosures: None 